\title{
Communication
}

\section{Speeding up nuclear magnetic resonance spectroscopy by the use of SMAll Recovery Times - SMART NMR}

\author{
Bruno Vitorge ${ }^{\mathrm{a}}$, Geoffrey Bodenhausen ${ }^{\mathrm{a}, \mathrm{b}}$, Philippe Pelupessy ${ }^{\mathrm{a}, *}$ \\ a Associé au CNRS UMR 7203 et à l'Université Pierre et Marie Curie, Département de Chimie, Ecole Normale Supérieure, 24 rue Lhomond, 75231 Paris Cedex 05, France \\ ${ }^{\mathrm{b}}$ Institut de Sciences et Ingénierie Chimiques, Ecole Polytechnique Fédérale de Lausanne, Batochime, 1015 Lausanne, Switzerland
}

\section{A R T I C L E I N F O}

\section{Article history:}

Received 25 June 2010

Revised 28 July 2010

Available online 1 August 2010

\section{Keywords:}

Fast NMR

$\cos Y$

TOCSY

Pulsed field gradients

Triple gradients

\begin{abstract}
A B S T R A C T
A drastic reduction of the time required for two-dimensional NMR experiments can be achieved by reducing or skipping the recovery delay between successive experiments. Novel SMAll Recovery Times (SMART) methods use orthogonal pulsed field gradients in three spatial directions to select the desired pathways and suppress interference effects. Two-dimensional spectra of dilute amino acids with concentrations as low as $2 \mathrm{mM}$ can be recorded in about $0.1 \mathrm{~s}$ per increment in the indirect domain.
\end{abstract}

(c) 2010 Elsevier Inc. All rights reserved.

\section{Introduction}

With the advent of ever-higher magnetic fields and improved instrumentation, nuclear magnetic resonance spectroscopy (NMR) is becoming increasingly sensitive. Very often the duration of a multidimensional experiment is primarily dictated by the need to obtain sufficient resolution in the indirect $\omega_{1}$ domain, rather than by the need to achieve a sufficient signal-to-noise ratio. Therefore, a myriad of methods have been developed to speed up the acquisition of multidimensional NMR experiments [1]. Most of the experimental time is taken by the recovery delay $t_{R D}$, i.e., the interval between two consecutive scans where the magnetization should return, at least in part, to equilibrium. Usually, it is recommended that $t_{R D}=\alpha T_{1}$ with $1<\alpha<5$, where $T_{1}$ is the longitudinal relaxation time. If the signals are sufficiently intense, one can reduce the recovery delay $t_{R D}$ by choosing $0<\alpha<1$, particularly if one uses selective excitation to avoid saturation of the protons that do not take part in the experiment and thus accelerate the return to equilibrium [2-4]. When $\alpha=0$ the magnetization recovers only during the time needed to record the free induction decay $t_{2}^{\max }$.

However, a reduction of $t_{R D}$ may have harmful consequences, in particular for small molecules with long relaxation times. Fig. 1 shows two spectra of a mixture of five amino acids, with relaxation times that vary in the range $1<T_{1}<8 \mathrm{~s}$, obtained by COrrelation

\footnotetext{
* Corresponding author. Fax: +33444323397.

E-mail address: philippe.pelupessy@ens.fr (P. Pelupessy).
}

SpectroscopY (COSY) [5], one with a $t_{R D}=4 \mathrm{~s}(0.5<\alpha<4)$ and the other with a $t_{R D}=10 \mathrm{~ms}(0.001<\alpha<0.01)$. Even in the latter case, the signal-to-noise ratio is sufficient, but a large number of artifacts, particularly in off-diagonal areas, make the spectrum difficult to interpret. These unwanted signals are due to interference of transverse and longitudinal magnetization components that 'leak' from one scan to another. In this sample, these signals appear when $t_{R D}<1 \mathrm{~s}$. The smaller $t_{R D}$, the larger the artifacts.

\section{Principles of the method}

Fig. 2 presents a general concept for the suppression of these artifacts, along with three specific examples. To quench residual single-quantum coherences (SQC's) and multiple-quantum coherences (MQC's) stemming from previous scans, a pulsed field gradient (PFG) $G_{Q}$ is applied. Note that zero-quantum coherences (ZQC's) cannot be suppressed in this manner. Thus, anti-phase SQC's should be dephased before they are converted into ZQC's. As usual, suitable PFG's are applied to select the desired coherence pathways during the sequence, but in order to prevent accidental refocusing, all gradients are chosen to be different from scan to scan. Changing the directions (rather than the amplitudes) of the gradients can achieve the desired effect. In this manner, losses due to translational diffusion do not differ from scan to scan. We shall use the prefix SMAll Recovery Time (SMART) to refer to such experiments. This method is reminiscent of work of Freeman and Hill [6], who in order to eliminate artifacts in one-dimensional Fourier transform NMR varied the recovery delay from scan to scan. 

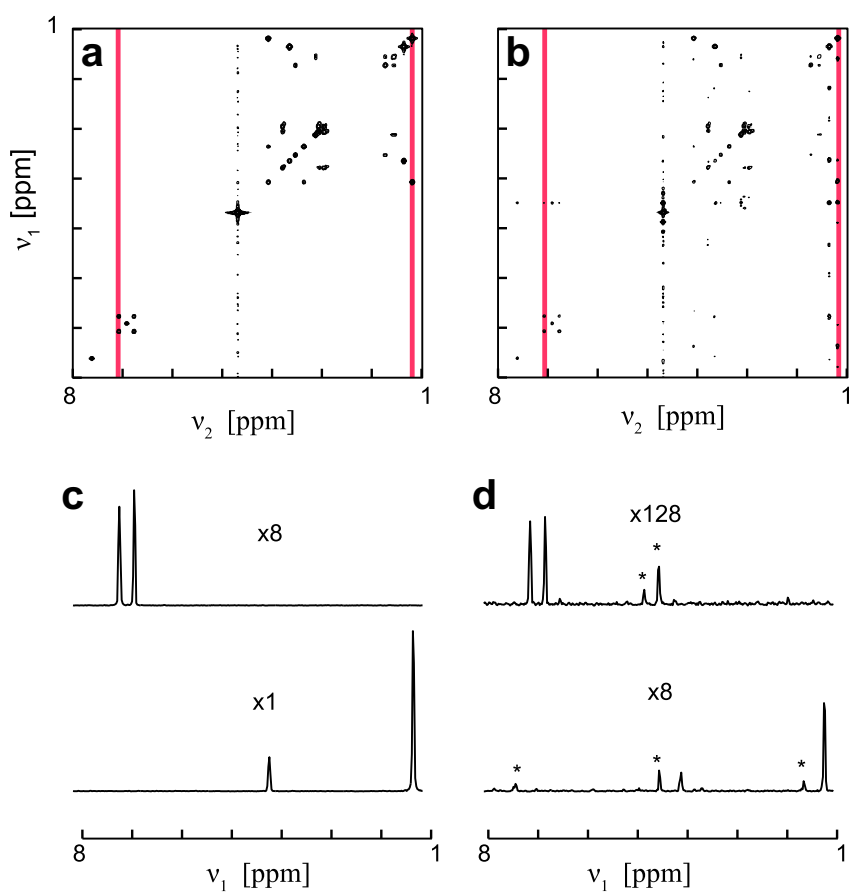

Fig. 1. (a) Classic (i.e., without the new SMART methodology) COSY spectrum of a mixture of $\sim 15 \mathrm{mM}$ Alanine, $\sim 7 \mathrm{mM}$ Arginine, $\sim 7 \mathrm{mM}$ Histidine, $\sim 10 \mathrm{mM}$ Threonine and $\sim 2 \mathrm{mM}$ Tyrosine in $\mathrm{D}_{2} \mathrm{O}$. The longitudinal relaxation times vary in the range $1<T_{1}<8 \mathrm{~s}$. The sequence uses gradients to select a single coherence transfer pathway [18] and does not give pure absorption peak shapes, so that an absolutevalue display must be used. In the detection interval, 1024 complex points were recorded with $t_{2}^{\max }=80 \mathrm{~ms}$. In the evolution interval, 512 increments were recorded with $t_{1}^{\max }=80 \mathrm{~ms}$. The recovery delay between subsequent transients was $t_{R D}=4 \mathrm{~s}\left(0.5<\alpha=t_{R D} / T_{1}<4\right)$, resulting in a total experimental time of about $36 \mathrm{~min}$. (b) Same experiment but with $t_{R D}=10 \mathrm{~ms}(0.001<\alpha<0.01)$, resulting in a total experimental time of about 70 s, i.e., a gain of a factor 30. Many undesired cross-peaks can be seen in this spectrum. The cross-sections (c) and (d) shown underneath correspond to columns at $v_{2} \approx 7.1 \mathrm{ppm}$ (upper rows) and $1.2 \mathrm{ppm}$ (bottom rows) (marked by red lines). The peaks marked with asterisks $(*)$ are artifacts. The bottom row corresponds to $10 \mathrm{mM}$ Threonine, while the top row stems from $2 \mathrm{mM}$ Tyrosine. Amplification factors are indicated above each spectrum.

\section{Experimental}

All experiments were performed on a sample of $\sim 15 \mathrm{mM}$ Alanine, $\sim 7 \mathrm{mM}$ Arginine, $\sim 7 \mathrm{mM}$ Histidine, $\sim 10 \mathrm{mM}$ Threonine and $\sim 2 \mathrm{mM}$ Tyrosine in $\mathrm{D}_{2} \mathrm{O}$ at a field strength of $14 \mathrm{~T}(600 \mathrm{MHz}$ proton frequency) and $298 \mathrm{~K}$. The spectrometer was equipped with a triple resonance inverse detection probe with pulsed field gradients along three axes. Before Fourier transformation the data sets of the $2 \mathrm{D}$ experiments were doubled in both dimensions by zero-filling and multiplied by a sine window function in the COSY experiments and by a cosine function in the other experiments.

\section{Results and discussion}

The advantages of SMART-COSY experiments are illustrated in Fig. 3. We generated a set of 163 different gradients $\boldsymbol{G}_{i}=a_{i} G_{x}+b_{i-}$ $G_{y}+c_{i} G_{z}$ with equal amplitudes $\left|\boldsymbol{G}_{i}\right|$ by varying the polar angles $\varphi_{i}=\arctan \left\{c_{i} /\left(a_{i}^{2}+b_{i}^{2}\right)^{1 / 2}\right\}$ and $\theta=\arcsin \left(a_{i} / b_{i}\right)$ to cover the northern half of the sphere uniformly, in such a way that the angle between any two directions is never smaller than $10^{\circ}$. In order to prevent accidental refocusing, we then ordered the gradients $G_{i}$ so as to maximize their orthogonality by maximizing the modules of the cross products $\left|\boldsymbol{G}_{i} \times \boldsymbol{G}_{j}\right|$ between pairs of gradients of the preceding 40 scans, giving decreasing weights $w_{i j}=(2 / 3)^{i-j}$ to more a

Quench Preparation Evolution Mixing Detection

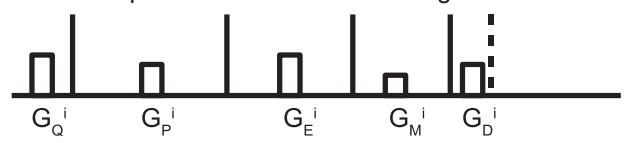

b

SMART-COSY
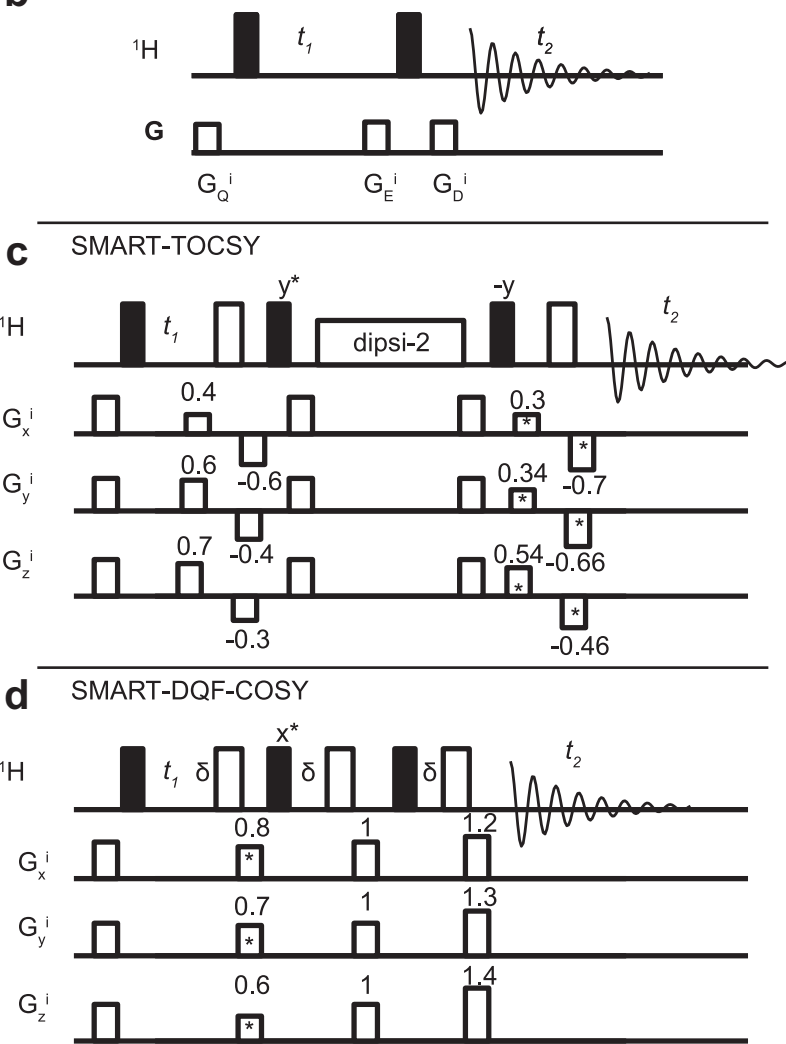

Fig. 2. (a) General scheme for SMART sequences. A typical two-dimensional experiment consists of a preparation interval, an evolution time, a mixing sequence, and a detection interval. A gradient $G_{Q}$ is used before the sequence starts to quench coherences stemming from the preceding scan. The other gradients serve to select the desired coherence transfer pathways. Often gradients are not needed during the preparation or mixing parts of the sequences. Multiple gradients may have to be applied at different stages (for concrete examples see below). It is important that the gradients differ from one scan to the next (hence the indices $i$ ). In order to maintain all experimental conditions as similar as possible from scan to scan, we chose to vary the directions of the gradients, while maintaining their amplitudes. (b) In SMART-COSY experiments, simple $90^{\circ}$ pulses (indicated by filled rectangles) are used for preparation and mixing. The quenching gradient $G_{Q}$ was chosen to be different in orientation as the coherence pathway selection gradients $G_{E}=G_{D}$. (c) In phase-sensitive $z$-filtered SMART-TOCSY [19], isotropic mixing is achieved by a DIPSI-2 pulse train [20]. (d) In phase-sensitive SMART-DQF-COSY, the complex signal is detected in the indirect $t_{1}$ dimension with the so-called Echo-Anti-Echo scheme, by inverting the phases of the pulses and the signs of the gradients marked with a star in every other scan in (c) and (d). The open rectangles indicate $180^{\circ}$ pulses. All pulses are along the $x$-axis except the two pulses on either side of the DIPSI-2 sequence. All gradients need to be different from one scan to another. For (b) $G_{E}=G_{D} \neq G_{Q}$. For (c) and (d) all gradients have different directions. The gradients that are marked with numbers have the same direction within one scan (but a different one from scan to scan), except for multiplication factors indicated by these numbers. The delay $\delta$ compensates for the duration of the gradient.

distant pairs, and excluding previously used gradients. Since for the latter gradients in the list there is less freedom of choice, only the first 123 of the ordered list of 163 gradients were used (for some larger molecules a set of only 20 different gradients was sufficient, however, the large set of 123 gradients always gave satisfactory spectra, regardless the size of the molecule). Listings are available from the authors on request. Such gradients can be generated either in conventional probes or in cryogenicaly cooled 

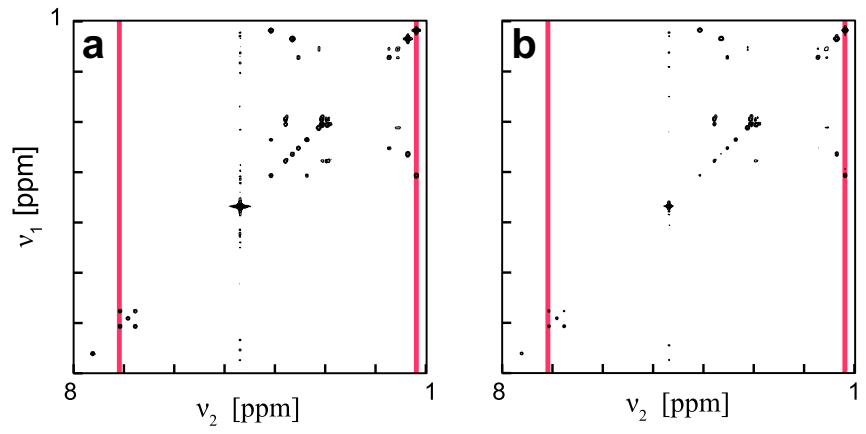

C
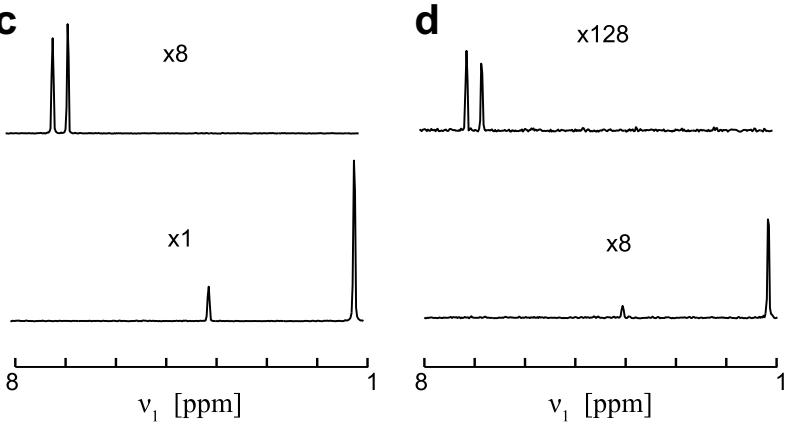

Fig. 3. Results of SMART-COSY with identical experimental conditions as in Fig. 1. (a) With $t_{R D}=4 \mathrm{~s}$, requiring about $2200 \mathrm{~s}$ (36 min). (b) With $t_{R D}=10 \mathrm{~ms}$, requiring about $70 \mathrm{~s}(1.1 \mathrm{~min})$. The bottom traces show that the artifacts marked by $*$ in Fig. 1 have been eliminated.

probes equipped with triple gradients [7]. The index $i$ of the gradients was incremented after the quenching gradient $G_{Q}$ and after the evolution and detection gradients $G_{E}$ and $G_{D}$. The two experiments in Fig. $3 \mathrm{a}$ and $\mathrm{b}$ have the same recovery delays $t_{R D}$ as in Fig. $1 \mathrm{a}$ and $\mathrm{b}$, but the undesired cross-peaks are largely suppressed. As with any method that does not allow full recovery of the longitudinal magnetization, the signal amplitudes are not proportional to the concentrations (i.e., not 'quantitative') when the relaxation rates vary from one nucleus to another.

In Fig. $4 \mathrm{~b}$, the method has been adapted for TOtal Correlation SpectroscopY [8] (SMART-TOCSY). Clearly the spectral quality has been considerably improved compared to the conventional experiment of Fig. 4a. One can double the signal intensity by eliminating the gradients before and after the mixing sequence. However, in this case the longitudinal magnetization that builds up during $t_{1}$ and ZQ coherences that could not be dephased before $t_{2}$ are present and can therefore affect the next increment. Surprisingly, in our sample this merely led to an increase of the $t_{1}$ noise and did not create any cross-peak artifacts (results not shown).

As a last example, we applied our methodology to DoubleQuantum Filtered (DQF) COSY. In this classic experiment, some artifacts are visible even if one uses a long delay $t_{R D}=4 \mathrm{~s}$. In Fig. 5, the improvement achieved with the SMART method using a short $t_{R D}=10 \mathrm{~ms}$ can be appreciated.

We are currently investigating whether varying the amplitudes of the gradients instead of their direction could be an option when PFG's are only available is a single direction. However, it would be very difficult if not impossible to prevent accidental refocusing when many gradients are used. Moreover, translational diffusion might attenuate the signals differently for distinct increments.

Consider an arbitrary sequence for 2D spectroscopy, where the average duration of each scan is $T=t_{R D}+t_{\text {seq }}+1 / 2 t_{1}^{\max }+t_{2}^{\max }$, where $t_{\text {seq }}$ is the length of the actual pulse sequence if $t_{1}=0$. If we assume that $t_{R D}=\alpha T_{1}$ in the traditional sequence, and $t_{R D}=0$ in the SMART version (i.e., $\alpha=0$ ), the gain in time is given by the ratio
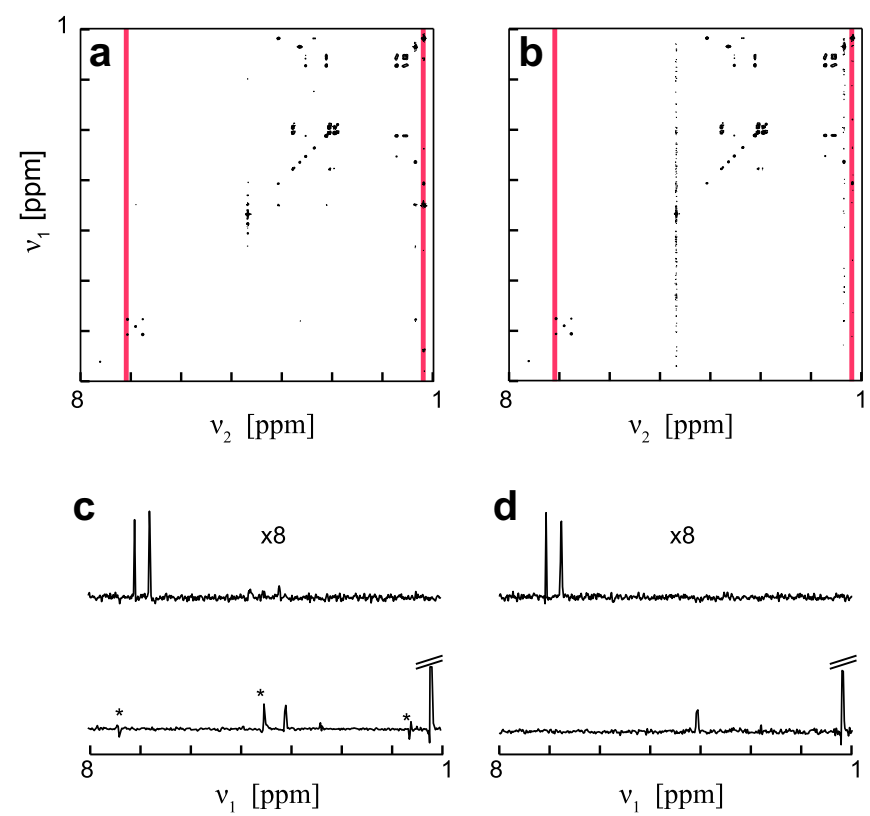

Fig. 4. Comparison of (a) normal TOCSY and (b) SMART-TOCSY, both obtained with a recovery delay $t_{R D}=10 \mathrm{~ms}$ and a mixing time $\tau_{\mathrm{m}}=40 \mathrm{~ms}$. In the evolution interval, 512 increments were recorded with $t_{1}^{\max }=80 \mathrm{~ms}$, each experiment requiring $190 \mathrm{~s}$. The columns below show the improvement in spectral quality. The peaks marked with asterisks $(*)$ are artifacts.

$\Gamma=\left(T-\alpha T_{1}\right) / T$

For example, for a TOCSY sequence with a mixing time $\tau_{\mathrm{m}}=40 \mathrm{~ms}, T_{1}=2 \mathrm{~s}, t_{1}^{\max }=t_{2}^{\max }=100 \mathrm{~ms}$, and $\alpha=1$, the ratio is $\Gamma \approx 0.1$, which amounts to a tenfold reduction in experimental time. The SMART method thus allows one to record highly resolved spectra in a reasonable amount of time.

We have given examples for three of the most commonly used routine homonuclear experiments applied to small molecules with

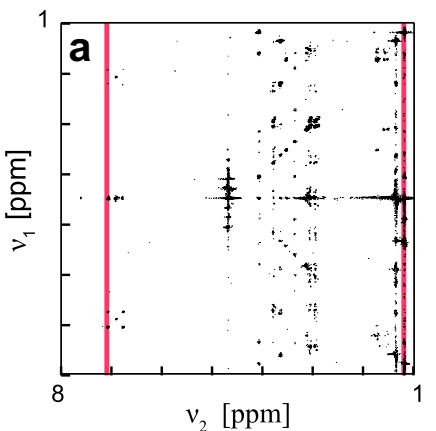

C

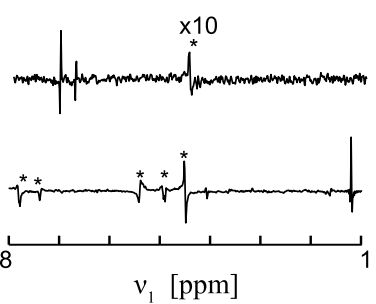

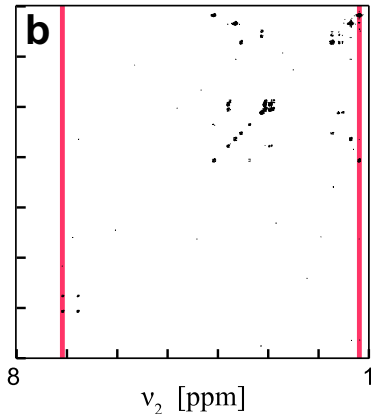

d

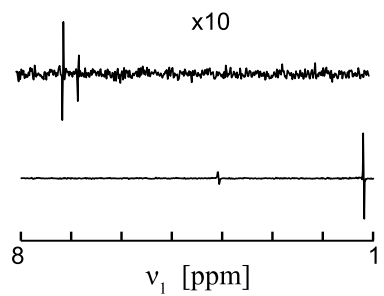

Fig. 5. Comparison of (a) normal DQF-COSY [21] and (b) SMART-DQF-COSY, both obtained with a recovery delay $t_{R D}=10 \mathrm{~ms}$, each experiment requiring $145 \mathrm{~s}$. The columns below show the improvement in spectral quality. The peaks marked with asterisks $(*)$ are artifacts. 
long relaxation times. Extensions to heteronuclear experiments should be feasible as long as the concentration is sufficient and the probe can handle high radio-frequency duty cycles, in particular if heteronuclear decoupling is required, although one could avoid decoupling by clever manipulations of spin states [22]. Extensions to more than two dimensions are currently under development. The importance of the artifacts strongly depends on the type of molecule and the duration of the recovery delay, but since varying the direction of the gradients from scan to scan does not have any adverse effects on the experiments, we recommend to always implement our scheme whenever triple axes gradients are available, no matter the recovery delay.

\section{Conclusions}

In this work we have presented a general method that allows a considerable reduction in experimental time of multidimensional NMR experiments, by as much as an order of magnitude. This technique does not require any special processing and is fully compatible with other time-saving methods such as filter diagonalization [9], covariance spectroscopy [10], linear prediction [11], nonCartesian sampling [12-14], spectral aliasing [15,16], and maximum entropy reconstruction [17]. The experiments require pulsed field gradients along three orthogonal axes. This appears to be a worthwhile investment for a much more efficient use of NMR spectrometers. We have demonstrated the utility of the method on a mixture of amino acids with concentrations as low as $2 \mathrm{mM}$ (routine experiments often use much higher concentrations). The use of a cryogenically cooled probe would allow reducing these concentrations by as much as a factor four. There appears to be no disadvantages associated with SMART pulse sequences, and we believe that almost any classical 2D experiment can be replaced by a SMART version, so that the recovery delay can be chosen to fit one's needs.

\section{Acknowledgments}

This work has been supported by the Integrated Infrastructure Initiative (I3) of the 6th Framework Program of the EC (Contract RII3-026145, EU-NMR), the Centre National pour la Recherche Scientifique (CNRS) of France and the Foundation Pierre-Gilles de Gennes (PGG).

\section{References}

[1] P. Schanda, Fast-pulsing longitudinal relaxation optimized techniques: enriching the toolbox of fast biomolecular NMR spectroscopy, Prog. Nucl. Magn. Reson. Spectrosc. 55 (2009) 238-265.

[2] K. Pervushin, B. Vogeli, A. Eletsky, Longitudinal H-1 relaxation optimization in TROSY NMR spectroscopy, J. Am. Chem. Soc. 124 (2002) 12898-12902.

[3] P. Schanda, B. Brutscher, Very fast two-dimensional NMR spectroscopy for real-time investigation of dynamic events in proteins on the time scale of seconds, J. Am. Chem. Soc. 127 (2005) 8014-8015.

[4] E. Lescop, P. Schanda, B. Brutscher, A set of BEST triple-resonance experiments for time-optimized protein resonance assignment, J. Magn. Reson. 187 (2007) 163-169.

[5] W.P. Aue, E. Bartholdi, R.R. Ernst, Two-dimensional spectroscopy - application to nuclear magnetic resonance, J. Chem. Phys. 64 (1976) 2229-2246.

[6] R. Freeman, H.D.W. Hill, Phase and intensity anomalies in Fourier transform NMR, J. Magn. Reson. 4 (1971) 366-383.

[7] R. Sarkar, D. Moskau, F. Ferrage, P.R. Vasos, G. Bodenhausen, Single or triple gradients?, J Magn. Reson. 193 (2008) 110-118.

[8] L. Braunschweiler, R.R. Ernst, Coherence transfer by isotropic mixing application to proton correlation spectroscopy, J. Magn. Reson. 53 (1983) 521-528.

[9] J. Chen, V.A. Mandelshtam, A.J. Shaka, Regularization of the two-dimensional filter diagonalization method: FDM2K, J. Magn. Reson. 146 (2000) 363-368.

[10] R. Bruschweiler, F. Zhang, Covariance nuclear magnetic resonance spectroscopy, J. Chem. Phys. 120 (2004) 5253-5260.

[11] P. Koehl, Linear prediction spectral analysis of NMR data, Prog. Nucl. Magn Reson. Spectrosc. 34 (1999) 257-299.

[12] E. Kupce, R. Freeman, Molecular structure from a single NMR experiment, J. Am. Chem. Soc. 130 (2008) 10788-10792.

[13] K. Kazimierczuk, A. Zawadzka, W. Kozminski, Optimization of random time domain sampling in multidimensional NMR, J. Magn. Reson. 192 (2008) 123130.

[14] H. Atreya, T. Szyperski, G-matrix Fourier transform NMR spectroscopy for complete protein resonance assignment, Proc. Natl. Acad. Sci. USA 101 (2004) 9642-9647.

[15] D. Jeannerat, High resolution in heteronuclear H-1-C-13 NMR experiments by optimizing spectral aliasing with one-dimensional carbon data, Magn. Reson. Chem. 41 (2003) 3-17.

[16] E. Lescop, P. Schanda, R. Rasia, B. Brutscher, Automated spectral compression for fast multidimensional NMR and increased time resolution in real-time NMR spectroscopy, J. Am. Chem. Soc. 129 (2007) 2756-2757.

[17] S. Hyberts, G. Heffron, N. Tarragona, K. Solanky, K. Edmonds, H. Luithardt, et al., Ultrahigh-resolution $\mathrm{H}-1-\mathrm{C}-13 \mathrm{HSQC}$ spectra of metabolite mixtures using nonlinear sampling and forward maximum entropy reconstruction, J. Am. Chem. Soc. 129 (2007) 5108-5116.

[18] P. Barker, R. Freeman, Pulsed field gradients in NMR. An alternative to phase cycling, J. Magn. Reson. 64 (1985) 334-338.

[19] K.E. Kövér, D. Uhrín, V.J. Hruby, Gradient- and sensitivity-enhanced TOCSY experiments, J. Magn. Reson. 130 (1998) 162-168.

[20] P.B. Barker, A.J. Shaka, R. Freeman, Homonuclear Hartmann-Hahn effects in broadband decoupling. J. Magn. Reson. 65 (1985) 535-539.

[21] R.E. Hurd, Gradient-enhanced spectroscopy, J. Magn. Reson. 87 (1990) 422 428.

[22] T. Kern, P. Schanda, B. Brutscher, Sensitivity-enhanced IPAP-SOFAST-HMQC for fast-pulsing 2D NMR with reduced radiofrequency load, J. Magn. Reson. 190 (2008) 333-338 